\title{
Imaging the Local Density of States of Optical Corrals
}

\author{
C. Chicanne, T. David, R. Quidant, J. C. Weeber, Y. Lacroute, E. Bourillot, and A. Dereux \\ Equipe Optique Submicronique, Laboratoire de Physique de l'Université de Bourgogne (CNRS UMR 5027), 9 avenue A. Savary, \\ F-21078 Dijon, France \\ G. Colas des Francs and C. Girard \\ Centre d'Elaboration des Matériaux et dEtudes Structurales (CNRS), 29 rue J. Marvig, F-31055 Toulouse, France
}

(Received 16 July 2001; published 14 February 2002)

\begin{abstract}
This paper reports the experimental observation, at optical frequencies, of the electromagnetic local density of states established by nanostructures corresponding to the recently introduced concept of optical corral [G. Colas des Francs et al., Phys. Rev. Lett. 86, 4950 (2001)]. The images obtained by a scanning near-field optical microscope under specific operational conditions are found in agreement with the theoretical maps of the optical local density of states. A clear functionality of detection by the scanning near-field optical microscope is thereby identified since the theoretical maps are computed without including any specific tip model.
\end{abstract}

DOI: $10.1103 /$ PhysRevLett.88.097402

PACS numbers: 78.67.Bf, 07.79.Fc, 78.68. +m

The density of states of elementary excitations is a basic concept underlying the description of a wide variety of physical phenomena. For example, the density of states of electromagnetic excitations at optical frequencies, not only photons but also polaritons, is well known to enter the theories of light matter (e.g., molecular lifetime change $[1,2])$ and van der Waals interactions. To describe the interaction between light and molecules, the dipolar approximation assumes that the incident optical electric field is spatially uniform at the molecular scale, even for long molecules. The formulation of the van der Waals interaction relies on the zero-point energy of the electromagnetic eigenmodes of the interacting objects. In both examples, the medium in which the molecules or the interacting objects are embedded is assumed to provide electromagnetic eigenmodes featuring a spatially uniform local density of states (LDOS). Not only in these contexts but also in many others, both tailoring the spectral properties or structuring the spatial distribution of the optical density of states are expected to lead to interesting physical effects [3]. Recent calculations of the optical LDOS [4] demonstrated that, close to nanostructures deposited on a surface, the full optical LDOS is dominated by subwavelength features: the weight of radiative eigenmodes is much smaller than the one of nonradiative modes.

At a given frequency, detecting the spatial variations of the optical LDOS is still lacking at the subwavelength, and, a fortiori, the molecular scale. To find a way to detect this optical LDOS, one may consider the analogy to electron physics. Specifically, in electron scanning tunneling microscopes (STM), for a weak coupling between tip and surface, the tunneling current is proportional to the value of the electron LDOS at the tip position [5]. Based on STM imaging of "quantum corrals," several impressive demonstrations of this property have been reported during the last decade [6-8]. The relation between tunnel current and electron LDOS led Carminati and Sáenz to general- ize Bardeen's formula for STM to the scattering of electromagnetic waves [9], thereby opening a way towards a unified approach of STM and scanning near-field optical microscopes (SNOM). On the basis of practical considerations about optical measurements in the near-field zone, Dereux et al. suggested that SNOM operating in illumination mode (where the light is incident through the probe) should detect the surface electromagnetic LDOS [10]. The present paper precisely deals with the experimental observation of the full optical LDOS using a SNOM in illumination mode.

While significant progress in the understanding of nearfield microscopes images in collection mode (where the light is incident through the substrate) have been achieved, the interpretation of SNOM images in illumination mode is an ongoing problem. This is related to the fact that, contrarily to electron STM where the tip design is well defined, the tips used in SNOM may be quite different between various setups, leading to different images. To find a satisfactory agreement with experimental data obtained in illumination mode requires then to solve the full scattering problem for each specific experimental setup. This imposes to include the tip, or at least a model of the tip, in the theoretical description. As far as one is concerned with the scattering problem, this approach is of course scientifically sound. However, to define any kind of functionality of measurement, it leads one to consider the difficult task of inverse scattering.

To reach our goal of detecting the optical LDOS, we have adopted a different, practical point of view. Instead of analyzing the scattering problem raised by any specific couple of experimental device and tip, we fixed the experimental setup and then tried systematically various tips designs in order to find which ones eventually lead to imaging the optical LDOS on a subwavelength scale. Following this point of view, the criterion for the searched functionality is then given by the agreement of the experimental 
images with theoretical optical LDOS maps, as computed without including any tip. As detailed in the literature (see references in [10]), the LDOS in an infinitesimal volume centered on $\mathbf{r}=(x, y, z)$ is related to the square moduli of the electric fields associated to all electromagnetic eigenmodes of angular frequency $\omega$. In the case of a continuous spectrum, it is conveniently deduced from the electric Green's dyadic, or electric susceptibility tensor, $\mathbf{G}$ of a system through (Im denotes the imaginary part, $k_{0}=\omega / c$, and $c$ the speed of light in vacuum)

$$
\rho(\mathbf{r}, \omega)=\frac{1}{4 \pi^{2} k_{0}^{2}} \operatorname{Im}\{\operatorname{Tr} \mathbf{G}(\mathbf{r}, \mathbf{r}, \omega)\}=\sum_{j=x, y, z} \rho_{j}(\mathbf{r}, \omega),
$$

where, due to the vector character of electromagnetic fields, we have defined the "partial" LDOS by:

$$
\rho_{j}(\mathbf{r}, \omega)=\frac{1}{4 \pi^{2} k_{0}^{2}} \operatorname{ImG}_{j j}(\mathbf{r}, \mathbf{r}, \omega) .
$$

For a system described by its dielectric function $\epsilon(\mathbf{r}, \omega)$, the LDOS is related to (but is not identified with) the pointlike dipolar light source entering the Dirac $\delta$-function source term of the following wave equation

$$
\begin{aligned}
& -\nabla \wedge \nabla \wedge \mathbf{G}\left(\mathbf{r}, \mathbf{r}^{\prime}, \omega\right)+k_{0}^{2} \epsilon(\mathbf{r}, \omega) \mathbf{G}\left(\mathbf{r}, \mathbf{r}^{\prime}, \omega\right) \\
= & -4 \pi k_{0}^{2} \mathbf{I} \delta\left(\mathbf{r}-\mathbf{r}^{\prime}\right),
\end{aligned}
$$

which defines the Green's dyadic of the system. Through the unit dyadic I, each partial LDOS is related to a given orientation $x, y$, or $z$ of the pointlike source. As detailed in previous works, one can find $\mathbf{G}\left(\mathbf{r}, \mathbf{r}^{\prime}, \omega\right)$ by solving numerically the self-consistent discretized Dyson's equation starting from the analytically known Green's dyadic of the flat surface [11,12].

The above summary of the LDOS concept makes clear that the adopted point of view aims at finding the experimental conditions such that, in practice, one can consider the probe tip as a pointlike dipole oscillating at the angular frequency $\omega$. At this point, one should stress that the LDOS should not be confused with the field radiated by the dipole facing the sample surface. The LDOS rather describes how the fact of scanning close to the surface drives this field radiated by the dipole through the substrate: depending on the tip position, scattering channels may turn out to be open or closed. In general, even if the field emitted forwardly in vacuum by many probe tips may resemble the one emitted by an ideal dipole, this does not imply that the nonradiative components established by any given tip approximate the ones of a dipole. To assess the dipolar character of these nonradiative components, we therefore used the experimental setup (Fig. 1) known as "forbidden light" SNOM [13,14]. This configuration allows a clean filtering of the Fourier components which are radiative in the air gap between the tip and the sample [15]: an asset to detect LDOS changes at the subwavelength scale. For the specific case of the excitation of surface plasmons, this device has already been studied theoretically from the point of view of scattering theory [16].
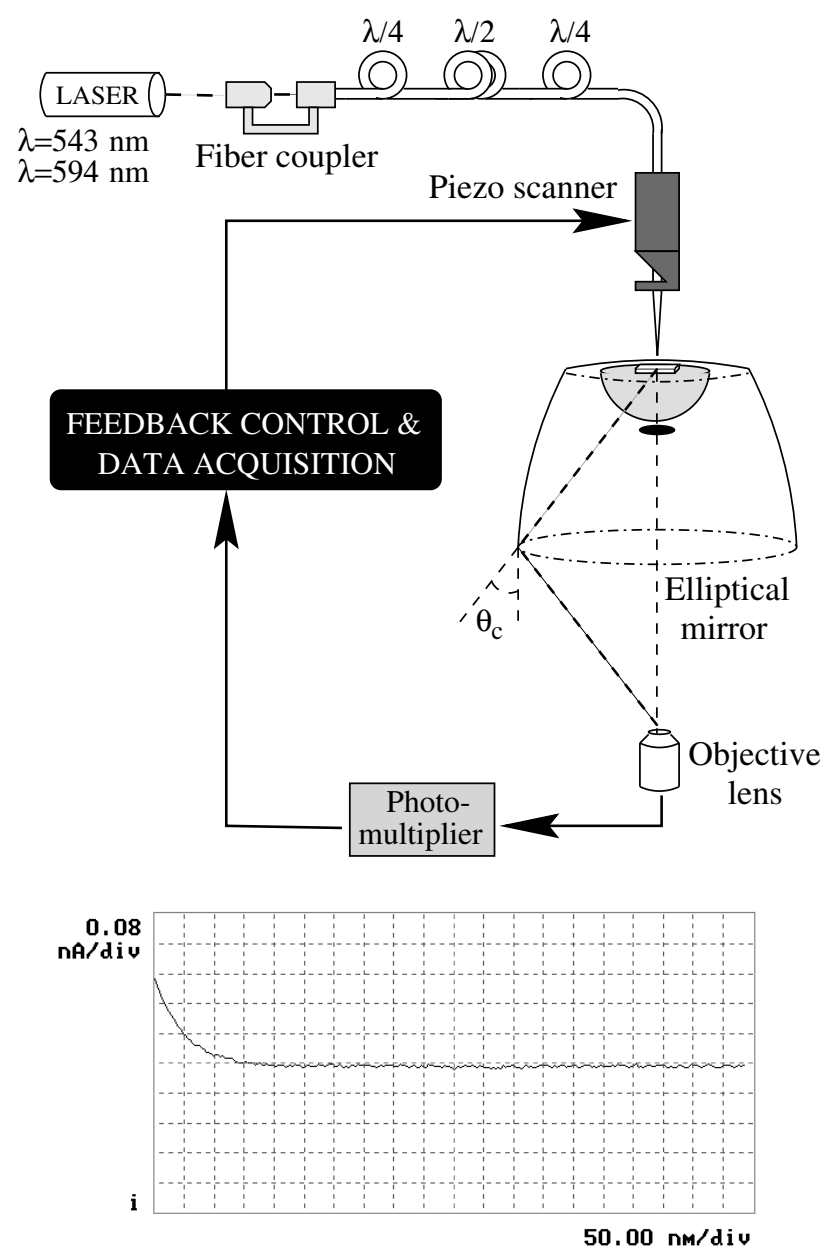

FIG. 1. Experimental SNOM setup. Typical curve of the intensity collected by the objective lens as a function of the tip-sample distance ( $z$ axis).

A linearly polarized laser (wavelength in vacuum $\lambda=$ $543 \mathrm{~nm}$ ) is injected in a tapered single-mode optical fiber with an apex radius of about $30 \mathrm{~nm}$. Subsequent coating with a metal enlarges this radius. The polarization of the field emerging from the tip is controlled by a standard device where three fiber loops approximate two quarter wave plates and a half wave plate. With this system, an elliptically polarized light with a typical ratio of $1 / 30$ between the minor and the major axis of polarization was measured in far field by rotating an analyzer in a plane perpendicular to the tip axis ( $x-y$ plane). The three fiber loops device enables us also to control the orientation of this major polarization axis. We use a hemispheric prism $(n=1.54)$ as a sample holder. When scanning the tip close to the sample surface, tunneling from the SNOM probe to the sample allows the conversion of waves which are evanescent in the gap into large wave vectors Fourier components in the prism. These large wave vectors emerge at angles $\theta$ greater than the critical angle for total reflexion in the prism $\theta_{c}$. The apex of the fiber is located at the focus of an elliptical mirror designed in such a way that only the radiations emerging at $\theta>\theta_{c}$ can be collected by an objective lens 
located at the second focus of the elliptical mirror. The tip is driven by the piezo actuator of a bioscope atomic force microscope (AFM) connected to a Nanoscope IIIA controller (both from Digital Instruments). Before scanning, the tip is approached along the $z$ axis, perpendicular to the sample surface (Fig. 1) until an exponential increase of the signal collected by the objective lens is detected in the vicinity of the sample surface (decay length of the order of $150 \mathrm{~nm}$ ). The results presented below used this signal to control the tip to sample distance in constant height mode.

Figure 2(a) is the AFM image of the nanostructures used as a test sample. They were fabricated by standard electron beam lithography. The resulting sample consists of gold nanoparticles $(100 \mathrm{~nm} \times 100 \mathrm{~nm} \times 50 \mathrm{~nm})$ deposited on the ITO substrate. The particles are arranged at $150 \mathrm{~nm}$ from each other in order to define the pattern of a $4 \mu \mathrm{m} \times 2 \mu \mathrm{m}$ corral stadium. The subwavelength optical LDOS changes of such optical analogies to electron quantum corrals [4] are well suited to test the capability of a SNOM to detect the optical LDOS dominated by nonradiative modes. Figures 2(b) 2(c), and 2(d) display the theoretical maps of the changes, relatively to the constant value in vacuum, of three partial LDOS calculated at the frequency of the laser. The subwavelength structuration of the LDOS appears clearly both inside and outside the sta- dium. Each partial LDOS displays a specific pattern inside the stadium: $\rho_{y}(\mathbf{r}, \omega)$ and $\rho_{z}(\mathbf{r}, \omega)$ feature two focal zones while $\rho_{x}(\mathbf{r}, \omega)$ leads to concentric replicas of the stadium shape. On top of the particles, $\rho_{x}(\mathbf{r}, \omega)$ and $\rho_{y}(\mathbf{r}, \omega)$ display decreasing values while $\rho_{z}(\mathbf{r}, \omega)$ shows much higher values.

Several tip designs (uncoated or coated with various thicknesses of different metals) were then used to scan the sample surface. Fiber tips coated with $20 \mathrm{~nm}$ of gold, without any resulting aperture at the apex, are found to lead to an encouraging agreement of experimental SNOM images [Figs. 3a and 3b] with the features of the theoretical partial LDOS maps [Fig. 2]. When the major polarization axis corresponds to a dipole source oriented along $x$ respectively (resp.) $y$ ], the experimental SNOM image displays inside the stadium the pattern typical for the $\rho_{x}(\mathbf{r}, \omega)$ [resp. $\left.\rho_{y}(\mathbf{r}, \omega)\right]$ partial LDOS map.

A more satisfactory agreement between theory and experiment is reached by refining the analysis as follows. As mentioned above, the orientation of the dipole source is achieved by rotating the major polarization axis. However, this reasoning in a $x-y$ plane located in the far-field zone takes into account only the radiative components of the field emitted by the tip such that it cannot provide any hint about a possible tilt of the dipole axis in the $z$ direction.
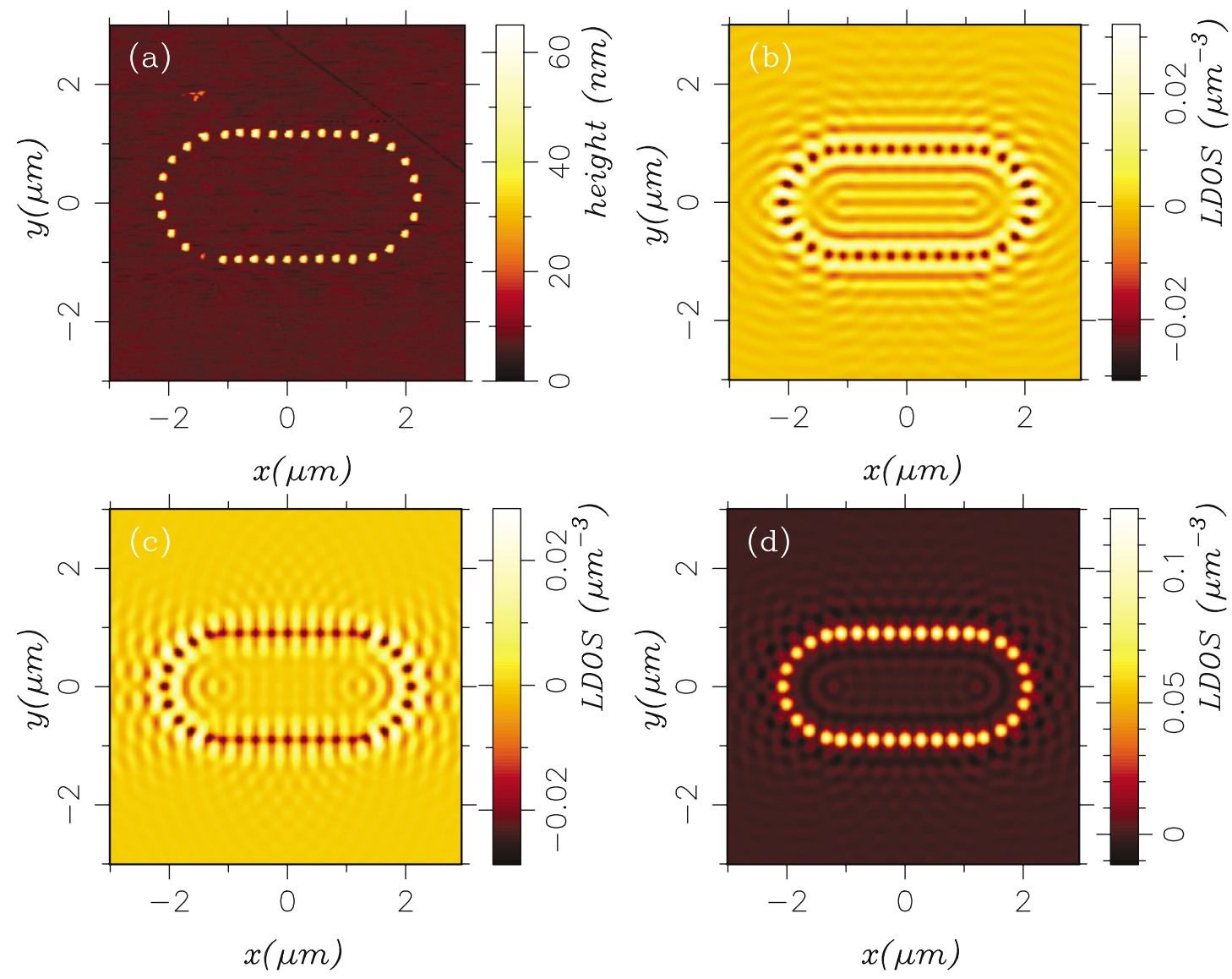

FIG. 2 (color). (a) AFM image of the topography of the sample. Relatively to the constant value in vacuum, theoretical partial LDOS changes computed close to the sample surface (height of calculation plane: $z=120 \mathrm{~nm}$ above the substrate); (b) $\rho_{x}(\mathbf{r}, \omega)$, (c) $\rho_{y}(\mathbf{r}, \omega)$, (d) $\rho_{z}(\mathbf{r}, \omega)$. 

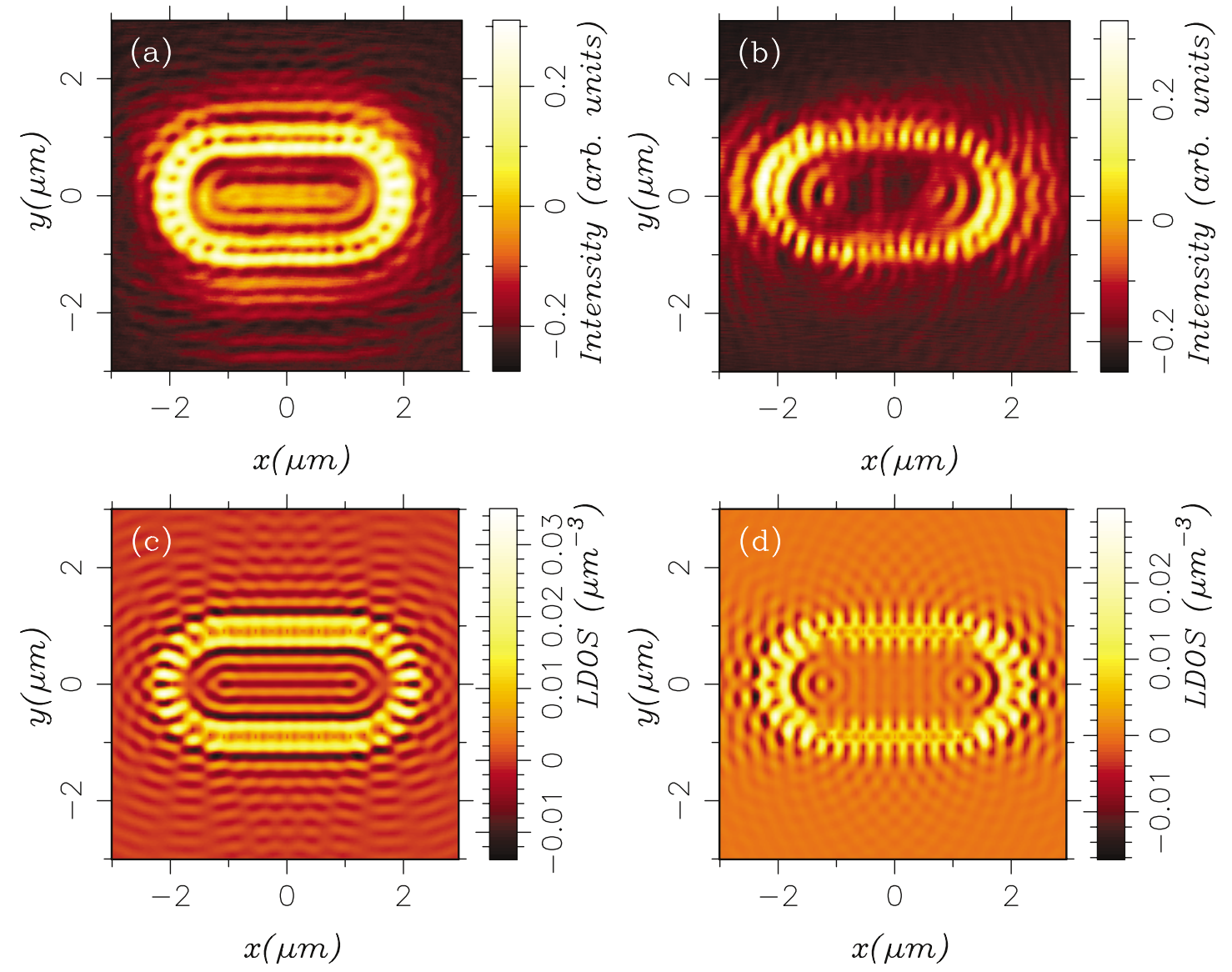

FIG. 3 (color). Experimental SNOM images recorded for two orthogonal polarization states corresponding to an orientation of the source dipole along (a) $x$ and (b) $y$. Linear combinations of the theoretical partial LDOS changes of Fig. 2: (c) $\rho_{x}(\mathbf{r}, \omega)+$ $0.25 \rho_{z}(\mathbf{r}, \omega)$, (d) $\rho_{y}(\mathbf{r}, \omega)+0.25 \rho_{z}(\mathbf{r}, \omega)$.

In the near-field zone, this $z$ component leads to a significant coupling. Therefore, if the source dipole axis has a $z$ and $y$ (resp. $x$ ) components, we expect that the SNOM signal mixes both partial LDOS $\rho_{z}$ and $\rho_{y}$ (resp. $\rho_{x}$ ). The present state of the art of tip manufacturing does not exactly control this tilt. Accounting for the $z$ component can be done by fitting the single parameter $\alpha$ which enters the two linear combinations $\alpha \rho_{z}+\rho_{x}$ and $\alpha \rho_{z}+\rho_{y}$. For both polarizations, the weight $\alpha=0.25$ provides convincing matches [Figs. $3 \mathrm{c}$ and $3 \mathrm{~d}$ ] with the experimental images [Figs. 3a and 3b].

In conclusion, for a specific type of local probe, a close relation between "forbidden light" SNOM signals and optical LDOS maps has been established experimentally. In molecular and chemical physics, this new kind of measurement suggests interesting perspectives of fundamental experiments relying on tailoring the spatial distribution of the optical local density of states at the nanometer scale.

This work was funded by the Regional Council of Burgundy and by the French Ministry for Education and Research through the program "Nanostructures et Fonctionalités."
[1] W. L. Barnes, J. Mod. Opt. 45, 661 (1998).

[2] P. Andrews and W. L. Barnes, Science 290, 785 (2000).

[3] S. John and J. Wang, Phys. Rev. B 43, 12772 (1991).

[4] G. Colas des Francs et al., Phys. Rev. Lett. 86, 4950 (2001).

[5] J. Tersoff and D. R. Hamann, Phys. Rev. B 31, 805 (1985).

[6] M. F. Crommie, C. P. Lutz, and D. M. Eigler, Nature (London) 363, 524 (1993).

[7] M. F. Crommie, C. P. Lutz, and D. M. Eigler, Science 262, 218 (1993).

[8] H.C. Manoharan, C. P. Lutz, and D. M. Eigler, Nature (London) 403, 512 (2000).

[9] R. Carminati and J. J. Sáenz, Phys. Rev. Lett. 84, 5156 (2000).

[10] A. Dereux, C. Girard, and J. C. Weeber, J. Chem. Phys. 112, 7775 (2000).

[11] O. J. F. Martin, C. Girard, and A. Dereux, Phys. Rev. Lett. 74, 526 (1995).

[12] C. Girard and A. Dereux, Rep. Prog. Phys. 59, 657 (1996).

[13] B. Hecht et al., Phys. Rev. Lett. 77, 1889 (1996).

[14] T. Huser et al., J. Opt. Soc. Am. A 16, 141 (1999).

[15] A. Dereux and D. Pohl, in Near Field Optics, edited by D. Pohl and D. Courjon, NATO ASI, Ser. E, Vol. 242.

[16] L. Novotny, B. Hecht, and D. Pohl, J. Appl. Phys. 81, 1798 (1997). 